\title{
The Effect of Exercise and Electrical Muscle Stimulation on the Architecture of the Vastus Medialis Oblique - The 'Empi’ Electrotherapy System
}

\author{
Zaib Hilal ${ }^{1}$, Claire J Robertson ${ }^{2}$, Alban Killingback ${ }^{3}$ and Philip J Adds ${ }^{1 *}$ \\ ${ }^{1}$ Institute of Medical and Biomedical Education (Anatomy), St. George's University of London, UK \\ ${ }^{2}$ Department of Medical Physics and Clinical Engineering, St. George's Healthcare NHS Trust, London, UK \\ ${ }^{3}$ Wimbledon Clinics, London, UK
}

*Corresponding author : Philip J Adds, Institute of Medical and Biomedical Education (Anatomy), St. George's University of London, Cranmer Terrace, London SW17 0RE, UK.

Received Date: October 03, 2018

Published Date: October 22, 2018

\section{Abstract}

Study Design: Asymptomatic volunteers underwent a vastus medialis oblique (VMO) muscle-strengthening programme, in both lower limbs. Neuro-muscular electrical stimulation (NMES) was applied to one limb, with the contralateral limb acting as the control.

Background: The usual first line treatment for patellofemoral pain is physiotherapy, targeting the vastus medialis oblique (VMO), with or without NMES. There is a lack of data on the effect of NMES on the architecture of the VMO. VMO.

Objectives: To investigate the effect of targeted exercises, with and without NMES, on the fiber angle and insertion level of the

Methods: Twenty-four asymptomatic female volunteers undertook a six-week muscle-strengthening programme targeting the VMO, on both lower limbs. An Empi electrical stimulator was used on one limb, with the other limb as the control. The VMO fiber angle with respect to the femoral axis, and insertion level on the medial border of the patella, before and after the exercise programme, were measured with ultrasound. Patellar length was measured, and the insertion ratio (insertion length/patella length*100) was calculated.

Results: Mean VMO fiber angle increase with and without NMES was 7.33 and 3.95, respectively. There was a significant increase in the insertion level in both limbs, but no significant difference between the stimulated and unstimulated limbs. There was a significant change in insertion ratio in the unstimulated limb. An inverse correlation was found between initial fiber angle and fiber angle change.

Conclusion: Electrical stimulation significantly enhances the effects of targeted exercises on the VMO fiber angle.

Keywords: Anatomy/lower extremities; Electrostimulation/electrotherapy; Patellofemoral joint

\section{Introduction}

The vastus medialis has (VM) been the object of much research. There is continuing controversy over whether it should be regarded as a single anatomical structure or if it consists of two separate muscles, the vastus medialis longus (VML) and the vastus medialis oblique (VMO), based on the difference between the muscle fiber angle with respect to the femoral axis [1,2]. The oblique angulation of the distal VMO fiber means that it provides a dynamic medial pull on the patella and is therefore implicated in patellar tracking [3]. A recent study has concluded that due to the electrophysiological difference of the VMO and VML, the two parts should be considered as separate muscles, especially for the purposes of knee rehabilitation [4]. The VM is also of interest due to its association with patellofemoral pain (PFP). PFP is a very common problem, particularly in young athletic females, resulting in anterior or retro patellar knee pain [5]. The prevalence of 
PFP is reported to vary from $8 \%$ to $40 \%$ [6]. While undoubtedly multifactorial in origin, PFP can be due to imbalances in the forces that control patellar tracking during flexion and extension of the knee, especially during strenuous activities that overload the joint, such as running [7]. VMO insufficiency, which can result in lateral patellar mal-tracking, is therefore thought to be implicated in the aetiology of PFP. First-line treatment for PFP usually consists of conservative physical therapy involving quadriceps-training exercises, specifically targeted at the VMO [8]. It has been shown that such exercises can increase the fiber angle of the VMO [9]. The increased strength and angulation of the muscle fibers increases the medial pull of the VMO on the patella and can help to alleviate the symptoms of this condition [10].

Electrotherapy is being increasingly used to aid the rehabilitation process in many musculoskeletal injuries. There is evidence to support the use of electrical muscle stimulation in the management of PFP [11] and a six-week electrotherapy programme with a cohort of 74 patients with PFP was found to give significant symptom relief [12]. Studies into the effect of exercise alongside electrical stimulation of the VMO have shown an increase in the force-generating capacity of the muscle alongside a decrease in pain in patients with PFP [13] and the use of electrical stimulation alongside physical therapy is now encouraged in the conservative management of PFP [14]. The study reported here aimed to evaluate the effects of exercise combined with electrical muscle stimulation on the fiber angle and insertion level of the VMO in asymptomatic individuals. Ultrasonography was used to allow an in-vivo investigation into the changes in muscle architecture following a six-week muscle-strengthening regime. Ultrasound is a safe, non-invasive imaging technique that has been used in many investigations into muscle architecture $[15,16]$. Measurement of VMO fiber angle and insertion level using ultrasound has been validated [28] and used in several previous investigations into the VMO in both symptomatic and asymptomatic individuals [9,17-19]. The aim of this study was to investigate the effect of the Empi electrical muscle stimulator on the architecture of the VMO following a six-week physiotherapy programme.

\section{Methods}

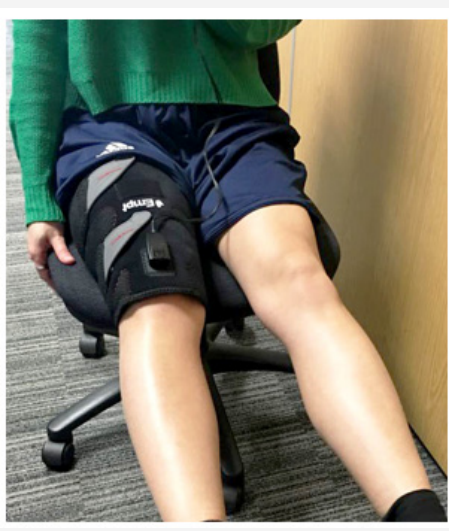

Figure 1: The Empi neuro-muscular electrical stimulation unit in use.

As PFP is more common in young women [5] an all-female cohort of twenty-five participants were recruited for this study. A power calculation indicated a required sample size of twenty-three to achieve statistical significance. All volunteers underwent an initial ultrasound scan, in which the parameters of VMO fiber angle and insertion level were recorded. The participants then undertook a six-week exercise programme, designed to target the VMO. Each was given an Empi muscle stimulator, which was assigned to either the left or right lower limb alternately (Figure 1).

Identical exercises were carried out on both limbs, one with the addition of the stimulator, and the other without. Three exercises were chosen, as there is evidence to suggest that adherence to exercise programmers decreases when more than 3 exercises are prescribed [20]. The participants were then scanned again. Non-athletic volunteers were recruited so that any observed changes would be attributable to the physiotherapy programme. Furthermore, previous studies have shown that there is a strong correlation between low initial VMO fiber angle, which is common among sedentary individuals [17] and the changes observed following physiotherapy [9].

Twenty-five volunteers were recruited to the study. One was assigned to the intra-rater reliability study, and so did not take part in the exercise programme. All gave informed written consent, and the study received ethical approval from the Institutional Research Ethics Committee. Participation in the study was based on the following inclusion criteria: age (18-25), female sex, no current or previous knee pain/pathology, no current or previous quadriceps pain/ pathology, no previous knee surgery, no lower limb deformities or abnormalities, and a Tegner Score $\leq 3$. The Tegner activity score is a method by which to numerically grade an individual's activity levels [21]. The average age of the participants was 19.54 years (range 18-22). The mean Tegner score was 2.58 (range 1-3). Sonographic images were taken using a Philips iU22 ultrasound machine, with a L17-5 linear array probe. Measurements were taken with Duratool digital callipers (accurate to $0.1 \mathrm{~mm}$ ) and a flexible goniometer.

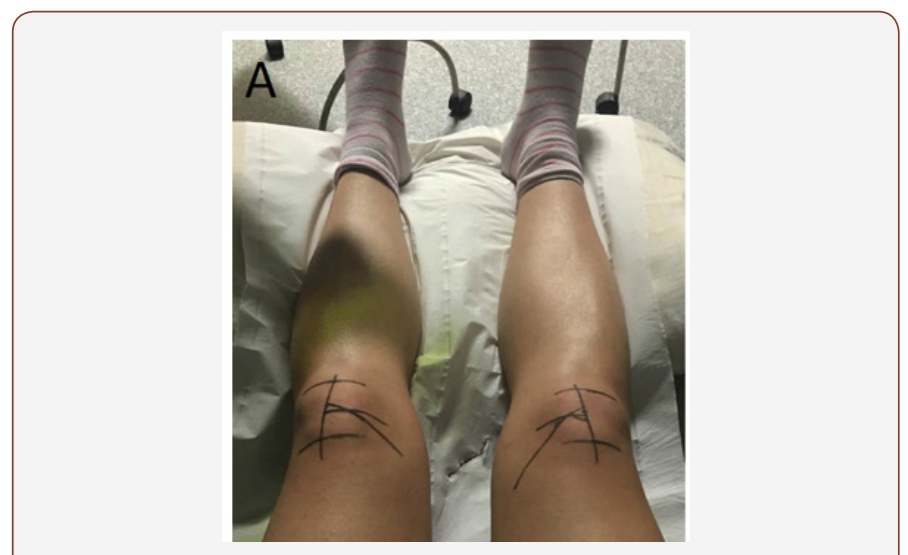

Figure 2A: The subjects lay supine with a pillow under the ankles.

All participants underwent an initial ultrasound scan. The methodology used in this study followed previously published studies in which ultrasound measurement of VMO architecture has been shown to be both valid and reliable. All scans and measurements were taken by the same operator throughout the study to eliminate inter-observer error. Participants lay supine on 
an examination couch, with a pillow under the ankles in order to keep both knees stationary (Figure 2A). The superior and inferior borders of the patella were palpated and marked. The patella length was measured using digital calipers. The mid-point of the patella was then located using the calipers and marked. The femoral axis was then marked, using a meter-rule placed between the subject's ASIS and the mid-point of the patella (Figure 2B).

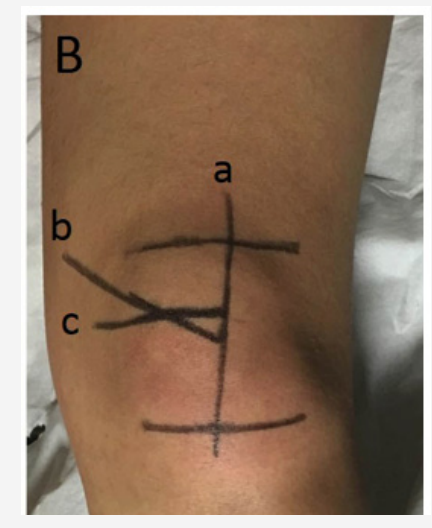

Figure 2B: The superior and inferior borders of the patella, femoral axis (a), VMO fiber angle (b), and insertion level (c) were marked on the skin.

The VMO fiber angle and insertion level were then determined with the ultrasound probe. Transmission gel was applied to the probe, which was then was placed on the distal VMO, and rotated until it was in line with the fibers. The position of the probe was marked. The probe was then moved distally until the muscle fibers could no longer be seen; this represents the insertion level of the VMO on the patella. The position of the probe was again marked (Figure 2B). The VMO fiber angle with respect to the femoral axis was measured using a flexible goniometer. The insertion level of the VMO was measured from the superior border of the patella. The insertion ratio was calculated by dividing this value by the total patella length and expressing the result as a percentage.

Following the initial scan, each volunteer was provided with an Empi Phoenix ${ }^{\mathrm{TM}}$ Neuromuscular Electrical Stimulator by DJO Global (Figure 1) set to program 2, which sends neuromuscular electrical stimulation (NMES) pulses cycling between a working frequency of $75 \mathrm{~Hz}$ for 4 seconds followed by a rest period of 10 seconds at a frequency of $4 \mathrm{~Hz}$. Participants then undertook a 6-week musclestrengthening program consisting of 3 sets of 3 different exercises to be performed on alternate days. Each set consisted of 12 repetitions, designed to cause light fatigue and induce muscular hypertrophy. The exercises were carried out on both limbs, with the Empi muscle stimulator on one limb, and the contralateral limb acting as a control. The stimulator also recorded the compliance of the volunteers, who had been made aware of this function. All participants were given full training in using the stimulator, and in the exercises they were required to follow. The three exercises in the physiotherapy program were: static quadriceps contractions while lying supine, knee extensions while sitting on the edge of a bed or chair, and 'mini-squats' from an upright position to $45^{\circ}$ of knee flexion. Participants were told to hold each position for 2 seconds, then rest for 3 seconds, which was synchronous with the timing of electrical stimulation. Upon completion of the 6-week exercise regime, a second ultrasound scan was carried out. The procedure was identical to the first scan. An intra-rater reliability study was carried out to determine the reliability of the recorded data. One participant, randomly selected from the cohort, did not take part in the physiotherapy programme, and was measured once a week over a period of 7 weeks. The parameters measured were exactly the same. A paired t-test was used to determine if the differences in fiber angle and insertion ratio before and after exercise was significant, and to determine if the difference between the stimulated and unstimulated limb was significant. For the intrarater reliability study, the coefficient of variation was calculated. Significance was set at $<0.05$. Statistical analysis was carried out using SPSS v 24.0 (IBM SPSS Statistics).

\section{Result}

\section{VMO fiber angle}

Table 1: VMO fibre angle change following physiotherapy with and without NMES. The difference between the + NMES and -NMES results was found to be significant $(P<0.001)$.

\begin{tabular}{|c|c|c|c|c|}
\hline & Mean & SD & Range & P-value \\
\hline $\begin{array}{c}\text { VMO fibre angle change }\left(^{\circ}\right)+ \\
\text { NMES }\end{array}$ & 7.33 & 1.84 & $4-10$ & $<0.001$ \\
\hline $\begin{array}{c}\text { VMO fibre angle change }\left(^{\circ}\right) \\
\text {-NMES }\end{array}$ & 3.95 & 0.91 & $2-6$ & $<0.001$ \\
\hline
\end{tabular}

\section{VMO insertion length}

Table 2: Changes in insertion length with and without NMES. The difference between the stimulated and unstimulated limb was found to be not significant $(P=0.231)$.

\begin{tabular}{|c|c|c|c|c|}
\hline & Mean & SD & Range & P-value \\
\hline $\begin{array}{c}\text { Insertion length change +NMES } \\
\text { (mm) }\end{array}$ & 0.38 & 0.75 & $-0.8-1.4$ & 0.02 \\
\hline $\begin{array}{c}\text { Insertion length change } \\
\text {-NMES (mm) }\end{array}$ & 0.74 & 1.13 & $-0.6-3.6$ & 0.004 \\
\hline
\end{tabular}

\section{Insertion ratio}

Table 3: Changes in insertion ratio (insertion length relative to patella length expressed as a percentage) with and without NMES. The difference between the +NMES and -NMES results was found to be not significant $(P=0.296)$.

\begin{tabular}{|c|c|c|c|c|}
\hline & Mean & SD & Range & P-value \\
\hline $\begin{array}{c}\text { Insertion ratio change +NMES } \\
(\%)\end{array}$ & 0.51 & 1.71 & $\begin{array}{c}-3.36- \\
4.86\end{array}$ & 0.156 \\
\hline $\begin{array}{c}\text { Insertion ratio change -NMES } \\
(\%)\end{array}$ & +1.21 & 2.39 & $\begin{array}{c}-2.61- \\
8.40\end{array}$ & 0.021 \\
\hline
\end{tabular}

\section{Intra-rater reliability study}

Table 4: Summary of the results of the intra-rater reliability study, indicating a high degree of reliability.

\begin{tabular}{|c|c|c|c|}
\hline & Mean & $\begin{array}{c}\text { Standard } \\
\text { Deviation (SD) }\end{array}$ & $\begin{array}{c}\text { Coefficient } \\
\text { of Variation }\end{array}$ \\
\hline Patella Length (mm) & 49.36 & 0.36 & 0.00729 \\
\hline Insertion Length (mm) & 19.43 & 0.21 & 0.01008 \\
\hline VMO Fiber Angle $\left(^{\circ}\right)$ & 73.86 & 0.38 & 0.00514 \\
\hline
\end{tabular}

\section{Relationship between initial VMO fiber angle and VMO fiber angle change}

There was a negative correlation between initial VMO fiber angle and fiber angle change. With NMES, the correlation was 
moderate $\left(\mathrm{R}^{2}=0.49\right)$; without NMES, the correlation was weaker $\left(\mathrm{R}^{2}=0.25\right)$ (Figure 3$)$.

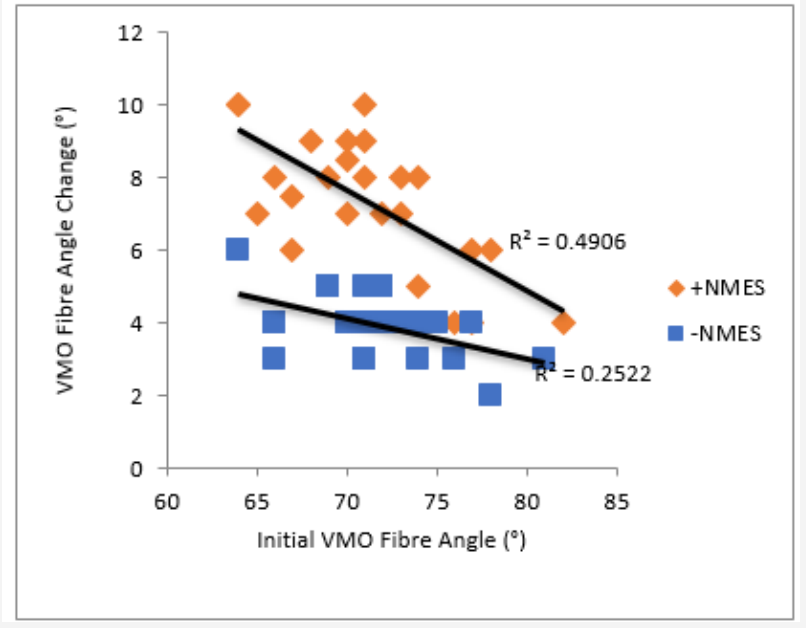

Figure 3: Initial VMO fiber angle vs VMO fiber angle change. There was a negative correlation between initial VMO fiber angle and fiber angle change. With NMES, there was a moderate correlation $\left(R^{2}=0.4906\right)$; without NMES, the correlation was weaker $\left(R^{2}=0.2522\right)$.

\section{Relationship between initial insertion length and change in insertion length}

With NMES, there was a moderate correlation between initial insertion length and the change in insertion length $\left(\mathrm{R}^{2}=0.33\right)$; without NMES, the correlation was found to be much weaker $\left(\mathrm{R}^{2}=0.034\right)$.

\section{Effect of compliance of volunteers and VMO fiber angle change}

The average compliance was $82.4 \%$ (range 50\% - 123.8\%) (100\% compliance was calculated to be equivalent to using the stimulator for a total of 210 minutes). Some participants completed the exercise regime more often than prescribed, and hence recorded compliance $>100 \%$. A strong positive correlation was found between compliance and VMO angle change in the + NMES limb $\left(\mathrm{R}^{2}=0.61871\right)$, and a moderate correlation in the -NMES limb $\left(\mathrm{R}^{2}=0.28724\right)$.

\section{Discussion}

Currently, in clinical practice, symptoms of PFPS may be managed by VM strengthening exercises [10] and significant improvements have been reported [22]. The management of PFPS requires long-term VMO strengthening, as the symptoms may return after cessation of the exercises [23]. Pennation angle has been shown to increase in response to resistance training [24] and the VMO fiber angle has been shown to increase following six weeks of targeted quadriceps exercises [9]. Electrically stimulated strength training has been shown to causes an increase in the maximal contractile force of a muscle [25]. The study reported here built upon these previous investigations, with the aim of investigating the combined effects of exercise with electrical stimulation on the architecture of the VMO. The Empi stimulator (randomly assigned to one lower limb, with the contralateral limb acting as a control) was set on program 2 (reinforcement), which uses NMES to stimulate the muscle into contracting. This is intended for use in physiotherapy for weak muscles (in conditions such as PFP), or where muscles are atrophied following injury or surgery [26]. The results presented here show a statistically significant increase in the fiber angle of the VMO in both stimulated and unstimulated limbs, with almost double the increase in the limb with the stimulator $\left(7.33^{\circ}+\mathrm{NMES}\right.$, compared to $3.95^{\circ}$-NMES). The increase brought about by the stimulator was found to be highly significant $(\mathrm{P}<0.001)$. A previous study has reported an increase of just over $5^{\circ}$ after a six-week physiotherapy regime [9] similar to the increase in the unstimulated limb reported here.

Our study also found an inverse correlation between initial VMO angle and the amount of change after the strengthening regime, with participants who had a lower initial fiber angle showing the greatest increase. This supports the suggestion by Khoshkhoo et al. that patients who might gain optimal benefit from physiotherapy could be easily screened by means of an ultrasound scan [9]. There was also a strong positive correlation between volunteer compliance and fiber angle, particularly in the +NMES group, suggesting that the most benefit from this type of therapy would be seen in patients with a low initial fiber angle, who used an NMES with high compliance. While there have been no other reports of research into the effect of NMES on the architecture of the VMO, the results reported here are supported by Werner et al. [27], who found that there was an increase in functionality of the VM in patients with PFP following transcutaneous electrical nerve stimulation (TENS). Garcia et al. [13] reported that the force generating capacity of the VM was significantly greater after NMES, and that this was associated with decreased pain following the treatment regime, probably as a result of correcting the imbalance between the VM and VL. Jan et al. [28] and Khoshkhoo et al. [9]. both report an increase in both insertion length and insertion ratio after exercise. While this study found a significant increase in insertion length, both with and without NMES, there was no significant difference between the two groups, and the increase was in fact greater in the -NMES limb. There was a significant difference in the insertion ratio in the -NMES limb, but not in the +NMES limb, with no significant difference between the two groups. Changes in insertion length and ratio are not well understood, and more research is needed in this area.

\section{Limitations}

The main limitation that has to be considered in this study is the recording of the compliance of the volunteers. While the Empi muscle stimulator automatically records usage time cumulatively in hours and minutes, it is not possible to determine on which days the machine was being used, and indeed whether it was actually being used while exercising or was just left switched on. There was, however, a high degree of correlation between compliance and VMO angle change, with no obvious outliers, suggesting that the compliance had been accurately recorded. As with all studies of this type, there may have been an element of subjectivity in recording the insertion levels and fiber angles. This was minimized by having 
the same investigator carrying out all the measurements, and the intra-rater reliability study suggests that the results have a high degree of reliability.

\section{Conclusion}

This study found that there was a significant increase in VMO fiber angle with NMES after a six-week programme of physiotherapy, and that the increase was significantly greater than in the limb without NMES. This study shows that the benefits of exercise, in terms of changes to the architecture of the VMO, can be greatly enhanced by the addition of NMES. This can be particularly helpful for elderly patients and those who may not be able to manage, or be less compliant with, strenuous amounts of exercise. Patients for whom this type of therapy would be of maximal benefit can be easily screened in clinic by an ultrasound scan.

\section{Acknowledgement}

The authors would like to thank Donjoy Ltd for the loan of the Empi muscle stimulators.

\section{References}

1. Nozic M, Mitchell J, De Klerk D (1997) A comparison of the proximal and distal parts of the vastus medialis muscle. Aust J Physiother 43(4): 277 281.

2. Skinner EJ, Adds PJ (2012) Vastus medialis: a reappraisal of VMO and VML. J Phys Ther Sci 24: 475-479.

3. Lin F, Wilson NA, Makhsous M, Press JM, Koh JL, et al. (2010) In vivo patellar tracking induced by individual quadriceps components in individuals with patellofemoral pain. J Biomech 43(2): 235-241.

4. Tenan MS, Hackney AC, Griffin L (2016) Entrainment of vastus medialis complex activity differs between genders. Muscle Nerve 53(4): 633-640.

5. Cook C, Hegedus E, Hawkins R, Scovell F, Wyland D (2011) Diagnostic accuracy and association to disability of clinical test findings associated with patellofemoral pain syndrome. Physiother Can 62(1): 17-24.

6. Peters JS, Tyson NL (2013) Proximal exercises are effective in treating patellofemoral pain syndrome: a systematic review. Int J Sports Phys Ther 8(5): 689-700.

7. Dixit S, Di Fiori J, Burton M, Mines B (2007) Management of patellofemoral pain syndrome. Am Fam Physician 75(2): 194-202.

8. Tang S, Chen C, Hsu R, Chou S, Hong W, et al. (2001) Vastus medialis obliquus and vastus lateralis activity in open and closed kinetic chain exercises in patients with patellofemoral pain syndrome: an electromyographic study. Arch Phys Med Rehab 82(10): 1441-1445.

9. Khoshkhoo M, Killingback A, Robertson CJ, Adds PJ (2016) The effect of exercise on vastus medialis oblique muscle architecture: an ultrasound investigation. Clin Anat 29(6): 752-758.

10. Mason M, Keays S, Newcombe P (2011) The effect of taping, quadriceps strengthening and stretching prescribed separately or combined on patellofemoral pain. Physiother Res Int 16(2): 109-119.

11. Bily W, Trimmel L, Modlin M, Kaider A, Kern H (2008) Training program and additional electric muscle stimulation for patellofemoral pain syndrome: a pilot study. Arch Phys Med Rehabil 89(7): 1230-1236.
12. Callaghan M, Oldham J (2004) Electric muscle stimulation of the quadriceps in the treatment of patellofemoral pain. Arch Phys Med Rehabil 85(6): 956-962.

13. Garcia FR, Azevedo FM, Alves N, Carvalho AC, Padovani CR, et al. (2010) Effects of electrical stimulation of vastus medialis obliquus muscle in patients with patellofemoral pain syndrome: an electromyographic analysis. Rev Bras Fisioter 14(6): 477-482.

14. Dos Santos RL, Souza ML, Dos Santos FA (2013) Neuromuscular electric stimulation in patellofemoral dysfunction: literature review. Acta Ortop Bras 21(1): 52-58.

15. Chino K, Akagi R, Dohi M, Takahashi H (2014) Measurement of muscle architecture concurrently with muscle hardness using ultrasound strain elastography. Acta Radiol 55(7): 833-839.

16. Lima K, Carneiro SP, De Souza Alves D, Peixinho C, Oliveira L (2015) Assessment of muscle architecture of the biceps femoris and vastus lateralis by ultrasound after a chronic stretching program. Clin J Sport Med 25(1): 55-60.

17. Benjafield A, Killingback A, Robertson C, Adds PJ (2015) An investigation into the architecture of the vastus medialis oblique muscle in athletic and sedentary individuals: an in vivo ultrasound study. Clin Anat 28(2): 262-268.

18. Engelina S, Antonios T, Robertson C, Killingback A, Adds PJ (2014) Ultrasound investigation of vastus medialis oblique muscle architecture: an in vivo study. Clin Anat 27(7): 1076-1084.

19. Jan M, Lin DH, Lin JJ, Lin CH, Cheng CK, et al. (2009) Differences in sonographic characteristics of the vastus medialis obliquus between patients with patellofemoral pain syndrome and healthy adults. Am J Sports Med 37(9): 1743-1749.

20. Henry K, Rosemond C, Eckert L (1999) Effect of number of home exercises on compliance and performance in adults over 65 years of age. Phys Ther 79(3): 270-277.

21. Tegner Y, Lysholm J (1985) Rating systems in the evaluation of knee ligament injuries. Clin Orthop Relat Res 198: 43-49.

22. Kaya D, Doral MN, Callaghan M (2012) How can we strengthen the quadriceps femoris in patients with patellofemoral pain syndrome? Muscles Ligaments Tendons J 2(1): 25-32.

23. Kannus P, Natri A, Paakkala T, Jarvinen M (1999) An outcome study of chronic patellofemoral pain syndrome. Seven-year follow-up of patients in a randomized, controlled trial. J Bone Joint Surg (Am) 81(3): 355-363.

24. Seynnes OR, de Boer M, Narici MV (2007) Early skeletal muscle hypertrophy and architectural changes in response to high-intensity resistance training. J Appl Physiol 102(1): 368-373.

25. Hortobagyi T, Maffiuletti NA (2011) Neural adaptations to electrical stimulation strength training. Eur J Appl Physiol 111(10): 2439-2449.

26. Walls R, McHugh G, O Gorman D, Moyna N, O Byrne J (2010) Effects of preoperative neuromuscular electrical stimulation on quadriceps strength and functional recovery in total knee arthroplasty. A pilot study. BMC Musculoskelet Disord 11: 119.

27. Werner S, Arvidsson H, Arvidsson I, Eriksson E (1993) Electrical stimulation of vastus medialis and stretching of lateral thigh muscles in patients with patello-femoral symptoms. Knee Surg Sports Traumatol Arthrosc 1(2): 85-92.

28. Engelina S, Robertson CJ, Moggridge J, Killingback A, Adds PJ (2014) Using ultrasound to measure the fibre angle of vastus medialis oblique: A cadaveric validation study. Knee 21(1): 107-111. 